\title{
Differences in long-term sedation agents used for the critically ill patients
}

\author{
Ahmed Ragab Zein ${ }^{1 *}$, Shahad Saad Albishi², Turki Nasser Alotaibi ${ }^{3}$, \\ Hidayah Dhiyaa Almanasif ${ }^{4}$, Mohammad Ibraheem Qashgry ${ }^{5}$, Saad Abdullah Al Holaibi ${ }^{6}$, \\ Lujain Darwish Raggam ${ }^{7}$, Shahad Abdullah Albogami ${ }^{8}$, Turki Salem Aljaza', \\ Nimah Jameel Dhafar ${ }^{5}$, Haifaa Immad Aldin AlAswad ${ }^{10}$
}

\author{
${ }^{1}$ Department of Intensive Care Unit, King Fahad General Hospital, Jeddah, Saudi Arabia \\ ${ }^{2}$ Department of Intensive Care Unit, King Faisal Hospital, Mecca, Saudi Arabia \\ ${ }^{3}$ Ministry of Health, Hail, Saudi Arabia \\ ${ }^{4}$ Department of Intensive Care Unit, Alkhafji General Hospital, Alkhafji, Saudi Arabia \\ ${ }^{5}$ College of Medicine, Umm Al-Qura University, Mecca, Saudi Arabia \\ ${ }^{6}$ College of Medicine, King Faisal University, Hofuf, Saudi Arabia \\ ${ }^{7}$ College of Medicine, Ibn Sina National College, Jeddah, Saudi Arabia \\ ${ }^{8}$ College of Medicine, Princess Nourah Bint Abdul Rahman University, Riyadh, Saudi Arabia \\ ${ }^{9}$ Department of Anaesthesia, King Khalid Hospital, Hail, Saudi Arabia \\ ${ }^{10}$ College of Medicine, Alfaisal University, Riyadh, Saudi Arabia
}

Received: 25 December 2021

Revised: 11 January 2022

Accepted: 12 January 2022

\section{*Correspondence:}

Dr. Ahmed Ragab Zein,

E-mail: ahmadragab@gmail.com

Copyright: (c) the author(s), publisher and licensee Medip Academy. This is an open-access article distributed under the terms of the Creative Commons Attribution Non-Commercial License, which permits unrestricted non-commercial use, distribution, and reproduction in any medium, provided the original work is properly cited.

\begin{abstract}
Sedative agents are commonly prescribed for critically ill patients admitted to the intensive care unit (ICU). The literature has reported many indications for using sedation for critically ill patients. These include reducing and managing high intracranial pressure, resolution of ventilator dyssynchrony, and decreasing agitation or anxiety. Different medications were reported in the literature as good sedatives for critically ill patients. Although very efficacious (benzodiazepines, propofol, and dexmedetomidine), many adverse events (as bradycardia, respiratory and myocardial depression, and hypotension) were reported as potential complications. The present literature review has discussed the potential differences and patients' outcomes after sedation with long-term modalities in the ICU. Overall, clinicians must critically consider balancing the harms and benefits of using sedatives for critically ill patients because of the potential complications encountered during these procedures. In addition, different sedatives were reported in the literature with variable efficacies and adverse events. For example, using dexmedetomidine and propofol has been more advantageous than using benzodiazepines, and some studies also favor dexmedetomidine. However, it should be noted that adverse events are still reported with all of these modalities. Therefore, the administration of long-term sedatives should follow a strict protocol to enhance patients' outcomes.
\end{abstract}

Keywords: ICU, Critically ill, Sedation, Anesthesia, Analgesics, Benzodiazepines 


\section{INTRODUCTION}

Sedative agents are commonly prescribed for critically ill patients admitted to the ICU. The literature has reported many indications for using sedation for critically ill patients. These include reducing and managing high intracranial pressure, resolution of ventilator dyssynchrony, and decreasing agitation or anxiety. Although many approaches were reported to optimize the process of sedation of the critically ill based on scoring systems and protocols, evidence shows that it is still a challenge and needs further optimization. ${ }^{1-4}$ Besides, although different analgesics were reported and validated in the literature, few were adequately investigated and studied in the ICU settings by randomized controlled trials (RCTs). ${ }^{5,6}$

Different medications were reported in the literature as good sedatives for critically ill patients. Although very efficacious (benzodiazepines, propofol, and dexmedetomidine), many adverse events (as bradycardia, respiratory and myocardial depression, and hypotension) were reported as potential complications. Studies indicate that their favorable outcomes and significance are remarkable variables between these sedatives. ${ }^{6-8}$ Accordingly, in the present study, we will discuss the potential differences between long-term sedatives used for critically ill patients.

\section{THE COMMON SEDATIVES}

\section{Propofol}

The current section will discuss the characteristics and adverse events of the most common sedative agents based on evidence from relevant studies. The first medication to be discussed would be propofol, validated in the literature and commonly used for critically ill patients. The main characteristics of the drug include the short duration and rapid onset of action. Moreover, it has been shown to have a short distribution half-life with a large volume of distribution. Moreover, it has been suggested that using the modality is favorable for patients with hepatic and renal dysfunction. Studies also indicate that emergence with propofol is more associated with metabolic clearance. ${ }^{9}$ However, it should be noted that some adverse effects are usually encountered when propofol is used. Hypertriglyceridemia, respiratory depression, myocardial depression, and vasodilatation-induced hypotension are the common one. The hypertriglyceridemic effect is usually observed with the propofol infusion syndrome (PRIS), which usually results secondary to altered hepatic metabolism of lipids or the intralipid carrier. ${ }^{10}$ Evidence indicates that PRIS is usually associated with severe rhabdomyolysis and lactic acidosis and can be predicted by some factors. ${ }^{10,11}$ These include prolonged infusion, critical illness, pediatric sedation, and increased dosage regimens. Accordingly, adequate care should be considered when dealing with these patients because of the highly reported mortality rate and the absence of adequate and specific treatment modalities. In this context, evidence shows that careful monitoring of the different vitals and associated variables of the high-risk patients is suggested to intervene against developing this syndrome and enhance their outcomes and prognosis. ${ }^{12}$

\section{Benzodiazepines}

These medications have been used for a long period for sedation in the ICU settings. Different medications in this classification of sedatives have been described, including diazepam, lorazepam, and midazolam. The literature discussed their validity, with favorable outcomes among the different studies. ${ }^{13}$ However, it has been reported that lorazepam can be associated with renal dysfunction and metabolic acidosis. Moreover, although many investigations have reported that these medications are widely used for sedation, other studies reported that many adverse events and complications could be associated with their use and administration within the ICU settings. These include increased time and length of ICU stay, mechanical ventilation, and increased incidence of brain dysfunction. ${ }^{14-16}$ Accordingly, more recent evidence indicates that using benzodiazepines for sedation in the ICU decreases based on these events. On the other hand, dexmedetomidine and propofol are more widely used in these settings as first-line agents. ${ }^{17,18}$ However, it should be noted that benzodiazepine medications are still the standard therapeutic modalities for managing patients with seizures and delirium.

\section{Dexmedetomidine}

This drug is an alpha-2 receptor agonist with favorable analgesia and sedation with no significant effects regarding respiratory depression. However, it should be noted that there is evidence indicating that the administration of this drug is usually associated with bradycardia and hypotension. ${ }^{19}$ Besides, bolus dosing of dexmedetomidine might be associated with stimulating post-junctional alpha-2 receptors located on the venous and arterial smooth muscles, cumulatively leading to the development of hypertension. Accordingly, it has been suggested that bolus dosing is not generally recommended for sedating critically ill patients. Another advantage of the drug is that there is no need to adjust the dose for renal patients. However, it has been shown that sedation with low doses is recommended for liver disorders because the liver usually metabolizes it. ${ }^{20}$

\section{Other sedatives}

Evidence shows that other pharmacological agents can be used in association with the sedative mentioned above modalities to improve their efficacy and reduce adverse events. For instance, ketamine has been described in these settings with favorable outcomes. It has been shown that it can be used in patients with severe degrees of burns to induce analgesia or potentiate the action of other 
analgesics, including opioids. ${ }^{21,22}$ However, its administration has been limited by the development of different adverse events. These include sympathetic stimulation, delirium, increased intracranial pressure, and induction of myocardial ischemia. However, more recent evidence suggests that it should be used in sedating patients with traumatic brain injuries because of its potential neuroprotective actions. ${ }^{23,24}$ Clonidine was also reported in the literature as a potential medication that can provide analgesia and sedation. It is commonly used for managing patients with withdrawal syndromes. ${ }^{25,26}$ It was also reported that facilitated extubation and reduced doses of opioids were also significant when clonidine was administered in patients admitted to the ICU. ${ }^{27}$ However, it should be noted that rebound hypertension was reported following using clonidine. Therefore, it has been suggested that quick and gradual discontinuation should be performed after administration. ${ }^{28}$ Using volatile anesthetics was also described in the literature. However, many disadvantages limit their use and administration for critically ill patients. ${ }^{29}$ On the other hand, recent evidence demonstrates that novel approaches were applied to overcome these potential limitations. Although using neuromuscular blockade has been described in this context, studies show that they are no longer considered for the critically ill because of complications. However, an RCT reported favorable outcomes among critically ill patients after being sedated with pharmacological paralysis. ${ }^{30}$ These findings still need further validation by future studies. ${ }^{31}$

\section{Differences in outcomes}

Many relevant RCTs were published in the current literature to investigate the safety and efficacy of various sedation agents widely used in intensive care settings. However, it should be noted that cumulative evidence from most of the included studies indicates that neither of the currently applied sedation agents significantly reduced the risk of mortality among critically ill patients. ${ }^{5,32-35}$ In addition, it has been shown that the time to perform tracheal extubation was significantly shorter with using propofol than using midazolam. However, evidence from these studies also demonstrated that midazolam has a higher risk of developing hypotension and hypertriglyceridemia. In another context, although a reduced risk of sedation-related delirium was significantly associated with dexmedetomidine, it has been shown that the drug is significantly associated with increasing the risk of hypotension and bradycardia. Currently, there is no sufficient evidence suggesting that using a single sedation agent can significantly reduce the risk of mortality and neurological complications. However, among patients with severe head injuries, it has been suggested that a transient decrease in cerebral perfusion pressure and increase in intracranial pressure is associated with using high bolus doses of opioids. ${ }^{36,37}$

The rates of adverse events were different among the currently reported sedative agents for the critically ill and are considered a key assessment for measuring the safety of these modalities. For instance, it has been shown that delirium has been significantly associated with using sedatives in ICUs. ${ }^{38}$ In addition, it has been shown that a low risk of developing delirium has been associated with using dexmedetomidine. On the other hand, a significantly high risk was associated with benzodiazepines (including lorazepam). Many burdens have subsequently been associated with the development of delirium in return. These include an increased risk of cognitive impairment, high costs, prolonged hospitalization, mechanical ventilation duration, and increased mortality risk. ${ }^{6}$ Accordingly, it has been suggested that future research focus on approaches to limit sedation to reduce the frequency of these events. In addition, sleep disturbances have been reported as common manifestations when admitted to the ICU. This has been associated with different factors. For example, mechanical ventilation has been reported to increase the risk of developing sleep disturbances during ICU admission. ${ }^{39}$ Although it has been suggested that sedative agents are usually administered to encounter these events, evidence shows that they do not yield in everyday sleep habits. Accordingly, previous studies reported that such actions are significantly associated with increased mortality, reduced muscle functions, and altered immune functions. ${ }^{6}$

Increased risk of developing ICU-related infections has been reported using sedation for critically ill patients. ${ }^{15,40,41}$ However, it should be noted that sepsis is one of the commonest causes for ICU admission and owes a huge mortality rate among admitted patients. ${ }^{42}$ In this context, a previous animal investigation reported that using sedative agents was significantly associated with influencing both adaptive and innate immunity. ${ }^{43}$ However, there is not enough data that would enable us to compare the different sedation agents used for critically ill patients regarding the impact of patient immunity. Evidence shows that more favorable immunological effects are significantly associated with dexmedetomidine in the ICU. ${ }^{33,41,43}$ Another adverse event that should be considered when comparing the safety of sedative agents is the accumulation of the drug and the duration taken to be aware and awake. It has been shown that the higher the accumulation rate in critically ill patients is usually associated with longer durations of infusion. Accordingly, using short-term sedation might be associated with more favorable events. However, there is insufficient data to compare the different sedative agents used with critically ill patients. ${ }^{6,7}$

Previous RCTs compared the efficacy and safety of benzodiazepines and propofol in achieving sedation and analgesia for critically ill patients. The authors reported reduced time spent on mechanical ventilation, decreased costs per patient, and increased duration at target arousal level were more significantly associated with propofol. ${ }^{16,44,45}$ This has been furtherly indicated in a previous meta-analysis, which showed that reduced 
duration of ICU stay was more significant with using short-time propofol than long-acting benzodiazepines. On the other hand, there was no significant difference when short-acting benzodiazepines were used. ${ }^{46}$ Other RCTs also found that dexmedetomidine had more significantly favorable results than benzodiazepines in terms of duration on mechanical ventilation and the incidence of hypertension and tachycardia. ${ }^{15,47}$ Worth mentioning, one RCT demonstrated that enhanced survival of critically ill patients was more significant with using dexmedetomidine than benzodiazepines. ${ }^{48}$ Comparing dexmedetomidine with propofol was also more significantly associated with less supplemental analgesia and reduced need for the administration of epinephrine and beta-blockers. ${ }^{33,49}$ In the same context, another RCT also compared the efficacy of propofol, dexmedetomidine, and midazolam among patients requiring mechanical ventilation for 24 hours. The authors reported that patients with dexmedetomidine spent less time on mechanical ventilation than patients in the midazolam group. Moreover, the extubation time was significantly shorter among patients in the dexmedetomidine than patients in the other two groups. However, it has been shown that there were no significant differences between the three groups regarding mortality and length of hospital and ICU stay. ${ }^{50}$

\section{CONCLUSION}

Overall, clinicians must critically consider balancing the harms and benefits of using sedatives for critically ill patients because of the potential complications encountered during these procedures. In addition, different sedatives were reported in the literature with variable efficacies and adverse events. For example, using dexmedetomidine and propofol has been more advantageous than using benzodiazepines, and some studies also favor dexmedetomidine. However, it should be noted that adverse events are still reported with all of these modalities. Therefore, the administration of longterm sedatives should follow a strict protocol to enhance patients' outcomes.

\section{Funding: No funding sources \\ Conflict of interest: None declared \\ Ethical approval: Not required}

\section{REFERENCES}

1. Devlin JW, Holbrook AM, Fuller HD. The effect of ICU sedation guidelines and pharmacist interventions on clinical outcomes and drug cost. Ann Pharmacotherapy. 1997;31(6):689-95.

2. Masica AL, Girard TD, Wilkinson GR. Clinical sedation scores as indicators of sedative and analgesic drug exposure in intensive care unit patients. The American journal of geriatric pharmacotherapy. 2007;5(3):218-31.

3. Brook AD, Ahrens TS, Schaiff R. Effect of a nursing-implemented sedation protocol on the duration of mechanical ventilation. Crit Care Med. 1999;27(12):2609-15.

4. Hansen-Flaschen JH, Brazinsky S, Basile C, Lanken PN. Use of sedating drugs and neuromuscular blocking agents in patients requiring mechanical ventilation for respiratory failure. A national survey. JAMA. 1991;266(20):2870-5.

5. Ostermann ME, Keenan SP, Seiferling RA, Sibbald WJ. Sedation in the intensive care unit: a systematic review. JAMA. 2000;283(11):1451-9.

6. Roberts DJ, Haroon B, Hall RI. Sedation for Critically Ill or Injured Adults in the Intensive Care Unit. Drugs. 2012;72(14):1881-916.

7. Karamchandani K, Dalal R, Patel J, Modgil P, Quintili A. Challenges in Sedation Management in Critically Ill Patients with COVID-19: a Brief Review. Curr Anesthesiol Rep. 2021;11(2):107-15.

8. Hughes CG, McGrane S, Pandharipande PP. Sedation in the intensive care setting. Clin Pharmacol. 2012;4:53-63.

9. Barr J, Egan TD, Sandoval NF. Propofol dosing regimens for ICU sedation based upon an integrated pharmacokinetic-pharmacodynamic model. Anesthesiology. 2001;95(2):324-33.

10. Diedrich DA, Brown DR. Analytic reviews: propofol infusion syndrome in the ICU. J Intensive Care Med. 2011;26(2):59-72.

11. Son PT, Reda A, Viet DC. Exchange transfusion in the management of critical pertussis in young infants: a case series. Vox Sanguinis. 2021;116(9):976-82.

12. Fudickar A, Bein B. Propofol infusion syndrome: update of clinical manifestation and pathophysiology. Minerva Anestesiologica. 2009;75(5):339-44.

13. Yahwak JA, Riker RR, Fraser GL, Subak-Sharpe S. Determination of a lorazepam dose threshold for using the osmol gap to monitor for propylene glycol toxicity. Pharmacotherapy. 2008;28(8):984-91.

14. Pandharipande P, Shintani A, Peterson J. Lorazepam is an independent risk factor for transitioning to delirium in intensive care unit patients. Anesthesiology. 2006;104(1):21-6.

15. Riker RR, Shehabi Y, Bokesch PM. Dexmedetomidine vs midazolam for sedation of critically ill patients: a randomized trial. JAMA. 2009;301(5):489-99.

16. Walder B, Elia N, Henzi I, Romand JR, Tramèr MR. A lack of evidence of superiority of propofol versus midazolam for sedation in mechanically ventilated critically ill patients: a qualitative and quantitative systematic review. Anesthesia Analgesia. 2001;92(4):975-83.

17. Mehta S, McCullagh I, Burry L. Current sedation practices: lessons learned from international surveys. Crit Care Clin. 2009;25(3):471-88.

18. Dibas M, Doheim MF, Ghozy S, Ros MH, El-Helw GO, Reda A. Incidence and survival rates and trends of skull Base chondrosarcoma: A Population-Based study. Clin Neurol Neurosur. 2020;198:106153.

19. Tan JA, Ho KM. Use of dexmedetomidine as a sedative and analgesic agent in critically ill adult 
patients: a meta-analysis. Intensive Care Med. 2010;36(6):926-39.

20. Maze M, Scarfini C, Cavaliere F. New agents for sedation in the intensive care unit. Cri Care Clin. 2001;17(4):881-97.

21. Laskowski K, Stirling A, McKay WP, Lim HJ. A systematic review of intravenous ketamine for postoperative analgesia. Can J Anaesth. 2011;58(10):911-23.

22. Nguyen TM, Huan VT, Reda A. Clinical features and outcomes of neonatal dengue at the Children's Hospital 1, Ho Chi Minh, Vietnam. J Clin virol. 2021;138:104758.

23. Martin J, Heymann A, Bäsell K. Evidence and consensus-based German guidelines for the management of analgesia, sedation and delirium in intensive care--short version. Ger Med Sci. 2010;8:Doc02.

24. Hirota K, Lambert DG. Ketamine: new uses for an old drug? Bri J Anaesthesia. 2011;107(2):123-6.

25. Cammarano WB, Pittet JF, Weitz S, Schlobohm RM, Marks JD. Acute withdrawal syndrome related to the administration of analgesic and sedative medications in adult intensive care unit patients. Crit Care Med. 1998;26(4):676-84.

26. Baumgartner GR, Rowen RC. Clonidine vs chlordiazepoxide in the management of acute alcohol withdrawal syndrome. Arch Internal Med. 1987;147(7):1223-6.

27. Liatsi D, Tsapas B, Pampori S, Tsagourias M, Pneumatikos I, Matamis D. Respiratory, metabolic and hemodynamic effects of clonidine in ventilated patients presenting with withdrawal syndrome. Intensive care Med. 2009;35(2):275-81.

28. Neusy AJ, Lowenstein J. Blood pressure and blood pressure variability following withdrawal of propranolol and clonidine. $\mathrm{J}$ Clin pharmacol. 1989;29(1):18-24.

29. Kong KL, Willatts SM, Prys-Roberts C. Isoflurane compared with midazolam for sedation in the intensive care unit. BMJ. 1989;298(6683):1277-80.

30. Papazian L, Forel JM, Gacouin A. Neuromuscular blockers in early acute respiratory distress syndrome. N Eng J Med. 2010;363(12):1107-16.

31. Thieu H, Bach Dat B, Nam NH. Antibiotic resistance of Helicobacter pylori infection in a children's hospital in Vietnam: prevalence and associated factors. Minerva Med. 2020;111(5):498-501.

32. Candiotti KA, Gan TJ, Young C. A randomized, open-label study of the safety and tolerability of fospropofol for patients requiring intubation and mechanical ventilation in the intensive care unit. Anesthesia Analgesia. 2011;113(3):550-6.

33. Venn RM, Grounds RM. Comparison between dexmedetomidine and propofol for sedation in the intensive care unit: patient and clinician perceptions. British J Anaesthesia. 2001;87(5):684-90.

34. Strøm T, Martinussen T, Toft P. A protocol of no sedation for critically ill patients receiving mechanical ventilation: a randomised trial. Lancet (London, England). 2010;375(9713):475-80.

35. Hall RI, MacLaren C, Smith MS. Light versus heavy sedation after cardiac surgery: myocardial ischemia and the stress response. Anesthesia Analgesia. 1997;85(5):971-8.

36. Roberts DJ, Hall RI, Kramer AH, Robertson HL, Gallagher CN, Zygun DA. Sedation for critically ill adults with severe traumatic brain injury: a systematic review of randomized controlled trials. Crit Care Med. 2011;39(12):2743-51.

37. El-Qushayri AE, Dahy A, Reda A. A closer look at the high burden of psychiatric disorders among healthcare workers in Egypt during the COVID-19 pandemic. Epidemiol Health. 2021;43:e2021045.

38. Ouimet S, Kavanagh BP, Gottfried SB, Skrobik Y. Incidence, risk factors and consequences of ICU delirium. Intensive Care Med. 2007;33(1):66-73.

39. Cooper AB, Thornley KS, Young GB, Slutsky AS, Stewart TE, Hanly PJ. Sleep in critically ill patients requiring mechanical ventilation. Chest. 2000;117(3):809-18.

40. Nseir S, Makris D, Mathieu D, Durocher A, Marquette $\mathrm{C}-\mathrm{H}$. Intensive care unit-acquired infection as a side effect of sedation. Critical Care. 2010;14(2):1-16.

41. Memiş D, Hekimoğlu S, Vatan I, Yandim T, Yüksel M, Süt N. Effects of midazolam and dexmedetomidine on inflammatory responses and gastric intramucosal $\mathrm{pH}$ to sepsis, in critically ill patients. Bri J Anaesthesia. 2007;98(4):550-2.

42. Vincent JL, Rello J, Marshall J. International study of the prevalence and outcomes of infection in intensive care units. JAMA. 2009;302(21):2323-9.

43. Sanders RD, Hussell T, Maze M. Sedation and immunomodulation. Anesthesiology Clin. 2011;29(4):687-706.

44. Cox CE, Reed SD, Govert JA. Economic evaluation of propofol and lorazepam for critically ill patients undergoing mechanical ventilation. Crit Care Med. 2008;36(3):706-14.

45. Barrientos-Vega R, Mar Sánchez-Soria M, MoralesGarcía C, Robas-Gómez A, Cuena-Boy R, AyensaRincon A. Prolonged sedation of critically ill patients with midazolam or propofol: impact on weaning and costs. Crit Care Med. 1997;25(1):33-40.

46. Ho KM, Ng JY. The use of propofol for medium and long-term sedation in critically ill adult patients: a meta-analysis. Intensive care medicine. 2008;34(11):1969-79.

47. Pandharipande PP, Pun BT, Herr DL. Effect of sedation with dexmedetomidine vs lorazepam on acute brain dysfunction in mechanically ventilated patients: the MENDS randomized controlled trial. JAMA. 2007;298(22):2644-53.

48. Pandharipande PP, Sanders RD, Girard TD. Effect of dexmedetomidine versus lorazepam on outcome in patients with sepsis: an a priori-designed analysis of the MENDS randomized controlled trial. Critical care (London, England). 2010;14(2):R38. 
49. Herr DL, Sum-Ping ST, England M. ICU sedation after coronary artery bypass graft surgery: dexmedetomidine-based versus propofol-based sedation regimens. J Cardiothorac Vasc Anesth. 2003;17(5):576-84.

50. Jakob SM, Ruokonen E, Grounds RM. Dexmedetomidine vs midazolam or propofol for sedation during prolonged mechanical ventilation: two randomized controlled trials. JAMA 2012;307(11):1151-60.

Cite this article as: Zein AR, Albishi SS, Alotaibi TN, Almanasif HD, Qashgry MI, Al Holaibi SA et al. Differences in long-term sedation agents used for the critically ill patients. Int J Community Med Public Health 2022;9:1023-8. 